\title{
A Game Theoretic Analysis of Distributed Power Control for Spread Spectrum Ad Hoc Networks
}

\author{
Jianwei Huang, Randall A. Berry, Michael L. Honig \\ Department of Electrical \& Computer Engineering, \\ Northwestern University, 2145 Sheridan Road, Evanston, IL 60208, USA \\ Email: \{jianweih, rberry, mh\}@ece.northwestern.edu
}

\begin{abstract}
We consider a distributed power control scheme in a Spread Spectrum (SS) wireless ad hoc network, in which each user announces a price that reflects his current interference level. Given these prices, we present an asynchronous distributed algorithm for updating power levels, and provide conditions under which this algorithm converges to an optimal power allocation. We relate this algorithm to myopic best response updates of a fictitious game, and characterize the algorithm's convergence using supermodular game theory.
\end{abstract}

\section{INTRODUCTION}

Power control is a basic technique for mitigating interference in wireless networks that enables efficient use of available spectrum. In ad hoc networks power control is complicated by the lack of centralized infrastructure, which necessitates the use of distributed approaches. In this paper, we consider distributed power control for SS ad hoc wireless networks, where each transmitter spreads its power over the available bandwidth and all interference is treated as noise. We focus on a model where the users receive a variable transmission rate that depends on their received signal-to-interference plus noise ratio (SINR). Our objective is to find distributed mechanisms for coordinating the users' power levels and optimizing overall performance, measured in terms of total network utility.

We consider a power control scheme in which the users exchange "price" signals that indicate the cost of received interference. Pricing mechanisms for allocating resources in networks has received considerable attention for both wire-line (e.g. [1]) and wireless networks (e.g. [2]-[4]). The problem here differs from much of the previous work because, due to interference, the users' objective functions are coupled with each other, and the overall network objective is not concave in the allocated resource (transmit power). Also, in most previous work, prices are Lagrange multipliers for some constrained resource such as power or bandwidth; here the prices reflect the interference or externalities among the users instead of a resource constraint. Our model is similar to [5], which also considers combined power and rate control for a SS ad hoc network. The power adaptation in [5] solves a similar problem to that considered here using gradient updates. Instead, we consider an approach based on supermodular game theory [6], which allows for different utility functions and appears to have faster convergence.

A variety of game-theoretic approaches have been applied to network resource allocation (e.g., [7]). Supermodular game theory, in particular, has been used to study power control in [8]-[10]. Our approach differs from those in that $(i)$ we focus on an ad hoc instead of a cellular network; $(i i)$ we consider a different functional form for the utilities, and (iii) we do not directly model the problem as a non-cooperative game. Instead, the users voluntarily cooperate with each other by exchanging interference information. We introduce a fictitious game and apply a strategy space transformation to view this algorithm as a supermodular game. Other work on power control in CDMA cellular and ad hoc networks includes [9][13]. In much of this work, a transmission is assumed to be successful only if a fixed minimum SINR requirement is met. This is true for fixed-rate communications. However, this is not the case for "elastic" data applications, in which users can adapt their transmission rate. In this paper, we focus on such rate-adaptive users, where the goal of power control is to maximize total network performance instead of guarantee interference margins for each user.

\section{SySTEM MODEL}

We consider a snap-shot of an ad hoc network with a set $\mathcal{K}=\{1, \ldots, K\}$ of distinct transmission pairs. Each pair consists of one dedicated transmitter and one dedicated receiver. This can represent a particular schedule of transmissions determined by an underlying routing and MAC layer protocol. We will use the terms "pair" and "user" interchangeably in the rest of the paper. Each user $k$ transmits an SS signal spread over the total bandwidth of $B \mathrm{~Hz}$. Over the time-period of interest we assume that the channel gains of the transmitterreceiver pairs are fixed. The channel gain between user $k$ 's transmitter and user $j$ 's receiver is denoted by $h_{k j}$. Note that in general $h_{k j} \neq h_{j k}$, since the latter represents the channel gain between user $j$ 's transmitter and user $k$ 's receiver. An example of a network with four users (pairs of nodes) is shown in Fig. 1.

The value of the transmission by user $k \in \mathcal{K}$ is characterized by a utility function $u_{k}\left(\gamma_{k}\right)$, which is an increasing and strictly concave function of the received SINR,

$$
\gamma_{k}(\boldsymbol{p})=\frac{p_{k} h_{k k}}{n_{0}+\frac{1}{B} \sum_{j \neq k} p_{j} h_{j k}},
$$






Fig. 1. SS ad hoc network of four users (pairs of nodes). $T_{k}$ and $R_{k}$ denote the transmitter and receiver of user $k$, respectively.

where $n_{0}$ is the background noise power and $\boldsymbol{p}=$ $\left(p_{1}, \cdots, p_{K}\right)$ is a vector of the users' transmission powers. The users' utility functions are coupled due to mutual interference. An example utility function is a logarithmic utility function $u_{k}\left(\gamma_{k}\right)=\theta_{k} \log \left(\gamma_{k}\right)$, where $\theta_{k}$ is a user dependent priority parameter. For low SINR, a user's rate is approximately linear in SINR; in this regime, the utility is therefore proportional to the logarithm of the rate. In the high SINR regime, the logarithmic utility approximates the Shannon capacity $\log \left(1+\gamma_{k}\right)$ weighted by parameter $\theta_{k}$.

The power control problem is to specify the vector of transmission powers, $\boldsymbol{p}$, where each user $k$ must also satisfy an individual transmission power constraint, $p_{k} \in \mathcal{P}_{k}=$ $\left[P_{k}^{\min }, P_{k}^{\max }\right]$, where $0 \leq P_{k}^{\min } \leq P_{k}^{\max }$. From a network perspective, the objective is to maximize the total utility summed over all users, i.e.,

$$
\max _{\left\{\boldsymbol{p}, p_{k} \in \mathcal{P}_{k}, \forall k \in \mathcal{K}\right\}} u_{\text {tot }}(\boldsymbol{p})=\sum_{k=1}^{K} u_{k}\left(\gamma_{k}(\boldsymbol{p})\right) .
$$

As a baseline, first consider the case where the users do not exchange any information and simply choose their transmission powers to maximize their individual utilities. As in [9], this can be modeled as a non-cooperative power control (NCPC) game, $G_{N C P C}=\left[\mathcal{K},\left\{\mathcal{P}_{k}\right\},\left\{u_{k}\right\}\right]$, where the players correspond to the users in $\mathcal{K}$; each player picks a strategy (transmission power) from the strategy set $\mathcal{P}_{k}=$ $\left[P_{k}^{\min }, P_{k}^{\max }\right]$ and receives a payoff $u_{k}\left(\gamma_{k}\right)$.

In this game, a power profile, $\boldsymbol{p}=\left(p_{1}, \ldots, p_{K}\right)$, is a vector of the users' transmission powers. The power profile of user $k$ 's opponents is defined to be $p_{-k}=\left(p_{1}, \ldots, p_{k-1}, p_{k+1}, \ldots, p_{K}\right)$, so that $\boldsymbol{p}=\left(p_{k} ; p_{-k}\right)$. User $k$ 's best response is

$$
\mathcal{B}_{k}\left(p_{-k}\right)=\arg \max _{p_{k} \in P_{k}} u_{k}\left(\gamma_{k}\left(p_{k}, p_{-k}\right)\right),
$$

i.e., the value $p_{k}$ that maximizes $u_{k}\left(\gamma_{k}\left(p_{k}, p_{-k}\right)\right)$ given a fixed $p_{-k}$. A power profile $\boldsymbol{p}^{*}$ is a Nash Equilibrium (NE) of the NCPC game if it is a fixed point of the users' best responses, i.e. $u_{k}\left(\gamma_{k}\left(p_{k}^{*} ; p_{-k}^{*}\right)\right) \geq u_{k}\left(\gamma_{k}\left(p_{k}^{\prime} ; p_{-k}^{*}\right)\right)$ for any $p_{k}^{\prime} \in \mathcal{P}_{k}$ and any user $k$.

Since each user's payoff $u_{k}\left(\gamma_{k}\left(p_{k}, p_{-k}\right)\right)$ is strictly increasing with $p_{k}$ for fixed $p_{-k}$, and there is no penalty for high transmission power as long as $p_{k} \in \mathcal{P}_{k}$, it is easy to verify that the unique NE of the NCPC game is $\boldsymbol{p}_{N C P C}^{*}=$ $\left(P_{1}^{\max }, \ldots, P_{K}^{\max }\right)$, i.e., each transmitter uses its maximum power. This solution can be far from the socially optimal solution, which maximizes the total utility.

Although $u_{k}\left(\gamma_{k}\right)$ is strictly concave in $\gamma_{k}$, the objective in Problem $\mathrm{P} 1$ may not be concave in $\boldsymbol{p}$. However, it is easy to verify that any local optimum, $\boldsymbol{p}^{*}=\left(p_{1}^{*}, \ldots, p_{K}^{*}\right)$, of this problem will be regular (see page 309 of [14]), and so must satisfy the following KKT necessary conditions:

Lemma 1 (KKT necessary conditions:): For any local optimal solution $\boldsymbol{p}^{*}$ of Problem P1, there exist unique Lagrange multiplier vectors $\boldsymbol{\lambda}^{*}=\left(\lambda_{1}^{*}, \ldots, \lambda_{K}^{*}\right)$ and $\boldsymbol{\mu}^{*}=\left(\mu_{1}^{*}, \ldots, \mu_{K}^{*}\right)$ such that for all $k \in \mathcal{K}$,

$$
\left.\left(\frac{\partial u_{k}\left(\gamma_{k}\left(p_{k}, p_{-k}\right)\right)}{\partial p_{k}}+\sum_{j \neq k} \frac{\partial u_{j}\left(\gamma_{j}\left(p_{j}, p_{-j}\right)\right)}{\partial p_{k}}\right)\right|_{\boldsymbol{p}=\boldsymbol{p}^{*}}=\lambda_{k}^{*}-\mu_{k}^{*}
$$

$\lambda_{k}^{*}\left(p_{k}^{*}-P_{k}^{\max }\right)=0, \mu_{k}^{*}\left(P_{k}^{\min }-p_{k}^{*}\right)=0$, and $\lambda_{k}^{*}, \mu_{k}^{*} \geq 0$.

Let

$$
\pi_{j}\left(p_{j}, p_{-j}\right)=-\frac{\partial u_{j}\left(\gamma_{j}\left(p_{j}, p_{-j}\right)\right)}{\partial I_{j}\left(p_{-j}\right)},
$$

where $I_{j}\left(p_{-j}\right)=\sum_{l \neq j} p_{l} h_{l j}$ is the total interference received by user $j$ (before bandwidth scaling). Here, $\pi_{j}\left(p_{j}, p_{-j}\right)$ is always nonnegative and represents user $j$ 's sensitivity to its current interference level, i.e., how much its utility would increase if the interference is decreased by one unit. This implicitly incorporates user $j$ 's utility information, i.e., the larger the utility (e.g., larger $\theta_{k}$ ) the larger is $\pi_{j}\left(p_{j}, p_{-j}\right)$. Using (3), condition (2), for all $k \in \mathcal{K}$, can be written as

$$
\left.\left(\frac{\partial u_{k}\left(\gamma_{k}\left(p_{k}, p_{-k}\right)\right)}{\partial p_{k}}\right)\right|_{\boldsymbol{p}=\boldsymbol{p}^{*}}-\sum_{j \neq k} \pi_{j}\left(p_{j}^{*}, p_{-j}^{*}\right) h_{k j}=\lambda_{k}^{*}-\mu_{k}^{*} \text {. }
$$

Assume each user $j$ announces $\pi_{j}\left(=\pi_{j}\left(p_{j}, p_{-j}\right)\right)$ as a price that he charges other users for generating interference, and that users are price takers (i.e. each user regards other users' prices as constants). Condition (4) is then a necessary and sufficient optimality condition for the problem in which each user $k$ specifies a power level $p_{k} \in \mathcal{P}_{k}$ to maximize the surplus function,

$$
s_{k}\left(p_{k}, p_{-k} ; \pi_{-k}\right)=u_{k}\left(\gamma_{k}\left(p_{k}, p_{-k}\right)\right)-p_{k} \sum_{j \neq k} \pi_{j} h_{k j},
$$

assuming fixed $p_{-k}$ and $\pi_{-k}$ (i.e., each user is a price taker and ignores any influence it may have on these prices). The surplus in (5) can be viewed as user $k$ 's utility minus a payment to the other users in the network due to the interference it generates. The payment is its transmit power times a weighted sum of other users' prices, with weights equal to the channel gains between user $k$ 's transmitter and the other users' receivers. This payment can be interpreted as a Pigovian tax [15].

\section{AsYNCHRONOUS Distributed PRICING (ADP) ALGORITHM}

The pricing interpretation of the KKT conditions motivates the following asynchronous distributed pricing (ADP) algo- 
rithm, in which users iteratively update their price announcements and transmit power decisions in an attempt to achieve a solution that satisfies Lemma 1 . For each $k \in \mathcal{K}$, define a power update function ${ }^{1}$

$$
\begin{aligned}
\mathcal{W}_{k}\left(p_{-k}, \pi_{-k}\right) & =\underset{\hat{p}_{k} \in \mathcal{P}_{k}}{\arg \max } s_{k}\left(\hat{p}_{k}, p_{-k} ; \pi_{-k}\right) \\
& =\left[\frac{p_{k}}{\gamma_{k}(\boldsymbol{p})} g_{k}\left(\frac{p_{k}\left(\sum_{j \neq k} \pi_{j} h_{k j}\right)}{\gamma_{k}(\boldsymbol{p})}\right)\right]_{P_{k}^{\min }}^{P_{k}^{\max }}
\end{aligned}
$$

and a price update function

$$
\mathcal{C}_{k}(\boldsymbol{p})=-\frac{\partial u_{k}\left(\gamma_{k}(\boldsymbol{p})\right)}{\partial I_{k}\left(p_{-k}\right)}=\frac{\partial u_{k}\left(\gamma_{k}(\boldsymbol{p})\right)}{\partial \gamma_{k}(\boldsymbol{p})} \frac{\left(\gamma_{k}(\boldsymbol{p})\right)^{2}}{B p_{k} h_{k k}},
$$

where $p_{k} / \gamma_{k}(\boldsymbol{p})$ is independent of $p_{k}$, and

$$
g_{k}(x)= \begin{cases}\infty, & 0 \leq x \leq u_{k}^{\prime}(\infty), \\ \left(u_{k}^{\prime}\right)^{-1}(x), & u_{k}^{\prime}(\infty)<x<u_{k}^{\prime}(0), \\ 0, & u_{k}^{\prime}(0) \leq x\end{cases}
$$

The ADP Algorithm is then specified as follows (where $t^{-}$ denotes the time immediately before $t$ ):

1.) Initialization: at $t=0$, each user $k \in \mathcal{K}$ chooses some initial power $p_{k}(0)$ and price $\pi_{k}(0)$.

2.) There are $2 K$ sets of infinite positive time instances $T_{k, p}$ and $T_{k, \pi}, k \in \mathcal{K}$, at which each user $k$ updates power $p_{k}(t)$ and price $\pi_{k}(t)$ as follows

$$
\begin{array}{ll}
p_{k}(t)=\mathcal{W}_{k}\left(p_{-k}\left(t^{-}\right), \pi_{-k}\left(t^{-}\right)\right), & \text {if } t \in T_{k, p}, \\
\pi_{k}(t)=\mathcal{C}_{k}\left(\boldsymbol{p}\left(t^{-}\right)\right), & \text {if } t \in T_{k, \pi} .
\end{array}
$$

Note that a user need not update his power and price at the same time. The asynchronous update is important in practice because it may be difficult to coordinate different users in the network to update their strategies in a synchronized manner.

This algorithm is distributed in nature not only because of the distributed generation of powers and prices by users, but also because each user needs to acquire limited information. Specifically, to implement the power and price updates, each user $k$ only needs to know: $(i)$ its own utility $u_{k}$, SINR $\gamma_{k}$ and channel gain $h_{k k},(i i)$ the "adjacent" channel gains $h_{k j}$ for $j \in \mathcal{K}$ and $j \neq k$, and (iii) the price profile $\pi$. By assumption each user knows its own utility. The SINR $\gamma_{k}$ and channel gain $h_{k k}$ can be measured at the receiver and fed back to the user. Measuring the adjacent channel gains $h_{k j}$ can be accomplished by having each receiver periodically broadcast a beacon; assuming reciprocity, the transmitters can then measure these channel gains. Note that the adjacent channel gains account for only $1 / K$ of the total channel gains in the network; each user does not need to know the other gains. The price information could also be periodically broadcast through this beacon. Each user announces a single price that is used by all interfering transmissions, so that the number of prices scales linearly with the size of the network. Also, numerical results suggest that there is little effect on

\footnotetext{
${ }^{1}$ Notation $[x]_{a}^{b}$ denotes $\max \{\min \{x, b\}, a\}$.
}

performance if users only convey their prices to "nearby" transmitters, i.e. those generating the strongest interference.

Denote the set of fixed points of the ADP algorithm by

$$
\mathcal{F}^{A D P}=\{(\boldsymbol{p}, \boldsymbol{\pi}) \mid(\boldsymbol{p}, \boldsymbol{\pi})=(\mathcal{W}(\boldsymbol{p}, \boldsymbol{\pi}), \mathcal{C}(\boldsymbol{p}))\},
$$

where $\mathcal{W}(\boldsymbol{p}, \boldsymbol{\pi})=\left(\mathcal{W}_{1}\left(p_{-1}, \pi_{-1}\right), \ldots, \mathcal{W}_{K}\left(p_{-K}, \pi_{-K}\right)\right)$ and $\mathcal{C}(\boldsymbol{p})=\left(\mathcal{C}_{1}(\boldsymbol{p}), \ldots, \mathcal{C}_{K}(\boldsymbol{p})\right)$.

Lemma 2: A power profile $\boldsymbol{p}^{*}$ satisfies the KKT conditions , of Problem P1 if and only if $\left(\boldsymbol{p}^{*}, \mathcal{C}\left(\boldsymbol{p}^{*}\right)\right) \in \mathcal{F}^{A D P}$.

The proof relies on the strict concavity of $u_{k}\left(\gamma_{k}\right)$. Furthermore, if the objective function of Problem P1 is strictly concave in $\boldsymbol{p}$ (or some monotonic transformation of $\boldsymbol{p}$ ), then the KKT conditions become necessary and sufficient for the unique optimal solution to Problem P1, and the ADP algorithm would reach that point if it converges. In general, Problem P1 may not be a concave maximization problem, so that the ADP algorithm may reach a locally optimal point (or saddle point) of Problem P1. ${ }^{2}$

\section{Convergence AnAlysis of ADP Algorithm}

In this section, we characterize the convergence of the ADP algorithm by viewing it in a game theoretic context. We first consider a game similar to the NCPC game, except now each player $k$ 's strategy includes specifying both a power $p_{k}$ and a price $\pi_{k}$ to maximize a payoff given by the surplus in (5). Since there is no penalty for user $k$ announcing a high price, it can be shown that each user's best response is to choose a large enough price to force all other users to stop transmission. This is certainly not a desirable outcome and suggests that the prices should be determined externally by another procedure.

Instead of the preceding game, we consider the following Fictitious Power-Price (FPP) control game: $G_{F P P}=$ $\left[\mathcal{F W} \cup \mathcal{F C},\left\{\mathcal{P}_{k}^{\mathcal{F W}}, \mathcal{P}_{k}^{\mathcal{F C}}\right\},\left\{s_{k}^{\mathcal{F} \mathcal{W}}, s_{k}^{\mathcal{F C}}\right\}\right]$, where the players are from the union of the sets $\mathcal{F W}$ and $\mathcal{F} \mathcal{C} . \mathcal{F W}=\{1, \ldots, K\}$ is the fictitious power player set, in which each player chooses a power $p_{k}$ from the strategy set $\mathcal{P}_{k}^{\mathcal{F W}}=\left[P_{k}^{\min }, P_{k}^{\max }\right]$. $\mathcal{F C}=\{1, \ldots, K\}$ is the fictitious price player set, in which each player chooses a price $\pi_{k}$ from the strategy set $\mathcal{P}_{k}^{\mathcal{F C}}=$ $\left[0, \bar{\pi}_{k}\right]$. Here $\bar{\pi}_{k}=\sup _{\boldsymbol{p}}\left(-\partial u_{k}\left(\gamma_{k}(\boldsymbol{p})\right) / \partial I_{k}\left(p_{-k}\right)\right)$, which could be infinite for some utility functions. A player $k \in \mathcal{F W}$ receives a payoff

$$
s_{k}^{\mathcal{F W}}\left(p_{k}, p_{-k} ; \pi_{-k}\right)=u_{k}\left(\gamma_{k}(\boldsymbol{p})\right)-\sum_{j \neq k} \pi_{j} h_{k j} p_{k},
$$

and a player $k \in \mathcal{F C}$ has a payoff

$$
s_{k}^{\mathcal{F C}}\left(\pi_{k} ; \boldsymbol{p}\right)=-\left(\pi_{k}+\frac{\partial u_{k}\left(\gamma_{k}(\boldsymbol{p})\right)}{\partial I_{k}\left(p_{-k}\right)}\right)^{2} .
$$

The payoff $s_{k}^{\mathcal{F C}}$ is defined so that the optimal strategy $\pi_{k}=$ $-\partial u_{k} / \partial I_{k}$. The players' best response functions are then

$$
\begin{aligned}
\mathcal{B}_{k}^{\mathcal{F W}}\left(p_{-k}, \pi_{-k}\right) & =\mathcal{W}_{k}\left(p_{-k}, \pi_{-k}\right), \forall k \in \mathcal{F} \mathcal{W}, \text { and11) } \\
\mathcal{B}_{k}^{\mathcal{F} \mathcal{C}}(\boldsymbol{p}) & =\mathcal{C}_{k}(\boldsymbol{p}), \forall k \in \mathcal{F C} .
\end{aligned}
$$

\footnotetext{
${ }^{2}$ Of course, no algorithm that relies only on first-order information can be guaranteed to converge to a global or even local optimum if the optimization problem is non-concave.
} 
In the FPP game, each user $k \in \mathcal{K}$ in the original ad hoc network is split into two fictitious players, one in set $\mathcal{F W}$ who controls power $p_{k}$ and the other one in set $\mathcal{F C}$ who controls price $\pi_{k}$. Although users in the network cooperate with each other by exchanging interference information (instead of choosing prices to maximize their surplus), each fictitious player in the FPP game is selfish and maximizes its own payoff function. In the rest of the paper, a "user" refers to one of the $K$ transmitter-receiver pairs in set $\mathcal{K}$, and a "player" refers to one of the $2 K$ fictitious players in the set $\mathcal{F W} \cup \mathcal{F C}$.

It follows from (11) and (12) that the ADP algorithm ((6) and $(7)$ ) can be interpreted as if the players in the FPP game employ an asynchronous myopic best response (MBS) updates, i.e. the players update their strategies according their best responses assuming the other users' strategies are fixed at the time of the updates. It is known that the fixed points of MBS updates are the same as the NEs of a game (see Lemma 4.2.1 in [6]).

Lemma 3: $\left(\boldsymbol{p}^{*}, \boldsymbol{\pi}^{*}\right) \in \mathcal{F}^{A D P}$ if and only if $\left(\boldsymbol{p}^{*}, \boldsymbol{\pi}^{*}\right)$ is a $\mathrm{NE}$ of the FPP game.

It follows that proving the convergence of MBS updates of the FPP game are sufficient to prove the convergence of the ADP algorithm to a solution of KKT conditions. We next analyze this convergence using supermodular game theory [6].

We first introduce some definitions ${ }^{3}$. A twice differentiable function $f$ has increasing differences between variables $(x, t)$ if $\partial^{2} f / \partial x \partial t \geq 0$ for any feasible $x$ and $t \cdot{ }^{4}$ A game $G=$ $\left[\mathcal{K},\left\{\mathcal{P}_{k}\right\},\left\{s_{k}\right\}\right]$ is supermodular if for each player $k \in \mathcal{K}$, the strategy space $\mathcal{P}_{k}$ is a nonempty and compact subset of $\mathbb{R}$, the payoff function $s_{k}$ is continuous in all players' strategies, and $s_{k}$ has increasing differences between player $k$ 's strategy and any other player's strategy. Several important properties of supermodular games are summarized in the following theorem.

Theorem 1: In a supermodular game $G=\left[\mathcal{K},\left\{\mathcal{P}_{k}\right\},\left\{s_{k}\right\}\right]$,

(a) There exists a NE. In case of multiple NEs, there is a smallest one and largest one (component-wise).

(b) If the users' best responses are single-valued functions, and each user $k$ starts from the smallest (largest) element of the strategy space $\mathcal{P}_{k}$ and uses MBS updates, then the strategies monotonically converge to the smallest (largest) NE.

(c) If each user $k$ starts from any feasible strategy and uses MBS updates, then the strategies will eventually lie in the set bounded component-wise by the smallest and largest NE. If there exists a unique NE, then the users' strategies globally converge to that NE from any choice of initial strategies.

Properties (a) follows from Lemma 4.2.1 and 4.2.2 in [6]; (b) follows from Theorem 1 of [10] and (c) can be shown by Theorem 8 in [16].

Next we show that by an appropriate strategy space transformation, certain instances of the FPP game are equivalent

\footnotetext{
${ }^{3}$ More general definitions related to supermodular games are given in [6].

${ }^{4}$ If we choose $x$ to maximize a twice differentiable function $f(x, t)$, then the first order condition gives $\partial f(x, t) /\left.\partial x\right|_{x=x^{*}}=0$, and the optimal value $x^{*}$ increases with $t$ if $\partial^{2} f / \partial x \partial t>0$.
}

to supermodular games, and so Theorem 1 applies. We first study a simple two-user network, then extend the results to a $K$-user network.

\section{A. Two-user networks}

Consider a network with only two users. There are four players in the corresponding FPP game, two in $\mathcal{F W}$ and two in $\mathcal{F C}$. First, we check whether the FPP game is a supermodular game. It is obvious that each user $k \in \mathcal{F} \mathcal{W}$ has a nonempty and compact strategy set, and so does each user $k \in \mathcal{F C}$ if $\bar{\pi}_{k}<\infty$. This bounded price restriction is not satisfied with some utilities such as $u_{k}\left(\gamma_{k}\right)=\theta_{k} \gamma_{k}^{\alpha} / \alpha$ with $\alpha \in[-1,0)$, since $\pi_{k}=\theta_{k} \gamma_{k}^{\alpha+1} /\left(p_{k} h_{k k} B\right)$ is not bounded as $p_{k} \rightarrow 0$. However, since no user would transmit with zero power at the $\mathrm{NE}$ (otherwise the payoff is $-\infty$ ), we can bound $\pi_{k}$ by a large number that would never be reached by the ADP algorithm. This would not impact any of the users' choices, and so not change any of these results.

The remaining increasing difference condition for the players' payoff functions does not hold with the original definition of strategies $(\boldsymbol{p}, \boldsymbol{\pi})$ in the FPP game. For example, from (9), $\partial s_{k}^{\mathcal{F} \mathcal{W}} / \partial p_{k} \partial \pi_{j}=-h_{k j}<0$ for any $j \neq k$, e.g. a higher price leads the other users to decrease their powers. However, if we define $\pi_{j}^{\prime}=-\pi_{j}$ and let each user $j \in \mathcal{F C}$ choose a $\pi_{j}^{\prime}$ from the strategy set $\left[-\bar{\pi}_{j}, 0\right]$, then $\partial s_{k}^{\mathcal{F} \mathcal{W}} / \partial p_{k} \partial \pi_{j}^{\prime}=h_{k j}>0$, i.e. $s_{k}^{\mathcal{F W}}$ has increasing differences between the strategies $\left(p_{k}, \pi_{j}^{\prime}\right)$ (or equivalently $\left(p_{j},-\pi_{j}\right)$ ). If we can redefine users' strategies so that each player $k$ 's payoff function has increasing differences between his transformed strategy and any other player's transformed strategy, then the transformed FPP game will be supermodular.

Let $\gamma_{k}^{\min }=\min \left\{\gamma_{k}(\boldsymbol{p}): p_{j} \in \mathcal{P}_{j}, \forall j \in \mathcal{K}\right\}$ and $\gamma_{k}^{\max }=$ $\max \left\{\gamma_{k}(\boldsymbol{p}): p_{j} \in \mathcal{P}_{j}, \forall j \in \mathcal{K}\right\}$ for all $k \in \mathcal{K}$. Also define $G_{k}\left(\gamma_{k}\right)=-\gamma_{k} u_{k}^{\prime \prime}\left(\gamma_{k}\right) / u_{k}^{\prime}\left(\gamma_{k}\right)$. Then, an increasing and strictly concave utility function $u_{k}\left(\gamma_{k}\right)$ is defined to be

- Type I if $G_{k}\left(\gamma_{k}\right) \in[1,2], \forall \gamma_{k} \in\left[\gamma_{k}^{\min }, \gamma_{k}^{\max }\right]$;

- Type II if $G_{k}\left(\gamma_{k}\right) \in(0,1], \forall \gamma_{k} \in\left[\gamma_{k}^{\min }, \gamma_{k}^{\max }\right]$.

The term $G_{k}\left(\gamma_{k}\right)$ is called the coefficient of relative risk aversion in economics [15] and measures the relative concaveness of $u_{k}\left(\gamma_{k}\right)$. Many common utility functions are either Type I or Type II. Examples of Type I utility functions include $u_{k}\left(\gamma_{k}\right)=\theta_{k} \log \left(\gamma_{k}\right)$ and $\theta_{k} \gamma_{k}^{\alpha} / \alpha$ with $\alpha \in[-1,0)$. Examples of Type II utility functions include $\theta_{k} \log \left(\gamma_{k}\right)$, $\theta_{k} \log \left(1+\gamma_{k}\right), 1-e^{-\theta_{k} \gamma_{k}}$ (with $\theta_{k}<n_{0} /\left(P_{k}^{\max } h_{k k}\right)$ ) and $\theta_{k} \gamma_{k}^{\alpha} / \alpha$ with $\alpha \in(0,1]$. The logarithmic utility function is both Type I and II.

A Type I utility function is "more concave" than a Type II one. Namely, an increase in one user's transmission power would induce the other users to increase their powers (i.e., $\partial^{2} s_{k}^{\mathcal{F W}} / \partial p_{k} \partial p_{j} \geq 0$ for $\left.j \neq k\right)$; a Type II utility would have the opposite effect (i.e., $\partial^{2} s_{k}^{\mathcal{F} \mathcal{W}} / \partial p_{k} \partial p_{j} \leq 0$ for $j \neq k$ ). The strategy spaces must be redefined in different ways for these two types of utility functions to satisfy the requirements of a supermodular game. 
Proposition 1: The FPP game for a two-user network is a supermodular game in the transformed strategies $\left(p_{1}, p_{2},-\pi_{1},-\pi_{2}\right)$ if both users have Type I utility functions.

Proposition 2: The FPP game for a two-user network is a supermodular game in the transformed strategies $\left(p_{1},-p_{2}, \pi_{1},-\pi_{2}\right)$ if both users have Type II utility functions.

The proof of these propositions consist of checking the increasing differences conditions for each player's payoff function. These results along with Theorem 1 enable us to characterize the convergence of the ADP algorithm. For example, if the two users have Type II utility functions (and $\left.\bar{\pi}_{1}, \bar{\pi}_{2}<\infty\right)$, then $\mathcal{F}^{A D P}$ is nonempty. In case of multiple fixed points, there exist two extreme ones $\left(\boldsymbol{p}^{L}, \boldsymbol{\pi}^{L}\right)$ and $\left(\boldsymbol{p}^{R}, \boldsymbol{\pi}^{R}\right)$, which are the smallest and largest fixed points in terms of the strategies $\left(p_{1},-p_{2}, \pi_{1},-\pi_{2}\right)$. That is, for any fixed point $\left(\boldsymbol{p}^{*}, \boldsymbol{\pi}^{*}\right), p_{1}^{*} \in\left[p_{1}^{L}, p_{1}^{R}\right]$ and $p_{2}^{*} \in\left[p_{2}^{R}, p_{2}^{L}\right]$. Similar relations hold for the prices. If users initialize $(\boldsymbol{p}(0), \boldsymbol{\pi}(0))$ at $\left(P_{1}^{\min }, P_{2}^{\max }, 0, \bar{\pi}_{2}\right)$ or $\left(P_{1}^{\max }, P_{2}^{\min }, \bar{\pi}_{1}, 0\right)$, the power and prices converge monotonically to $\left(\boldsymbol{p}^{L}, \boldsymbol{\pi}^{L}\right)$ or $\left(\boldsymbol{p}^{R}, \boldsymbol{\pi}^{R}\right)$, respectively. If users start from arbitrary initial power and prices, then the strategies will eventually lie in the space bounded by $\left(\boldsymbol{p}^{L}, \boldsymbol{\pi}^{L}\right)$ and $\left(\boldsymbol{p}^{R}, \boldsymbol{\pi}^{R}\right)$. Similar arguments can be made with Type I utility functions with the corresponding strategy transformation. Transforming the FPP game into a supermodular game shows that the ADP algorithm is a stable feedback mechanism in the sense that the changes in powers and prices reinforce each other until a fixed point is reached. Convergence of the transmit powers under both types of utilities is illustrated in Fig. 2.
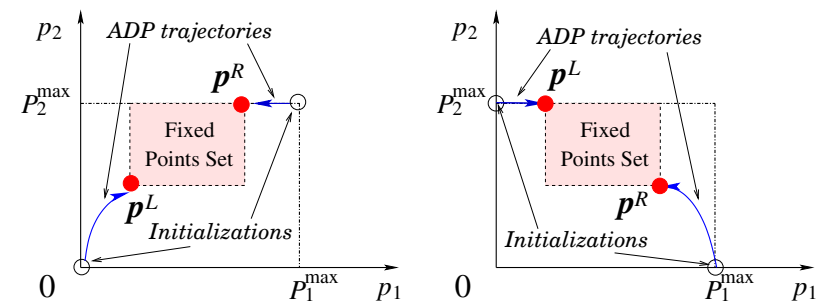

Fig. 2. A two-user network with Type I (left) or Type II (right) utility functions. ADP trajectories illustrate the monotonic convergence of the transmit powers. (Convergence of the prices is not shown here.) Here we assume $P_{1}^{\min }=P_{2}^{\min }=0$.

\section{B. K-user Networks}

Proposition 1 can be easily generalized to a network with $K>2$ users:

Corollary 1: For a $K$-user network, if all users have Type I utilities, then $G_{F P P}$ is supermodular in the transformed strategies $(\boldsymbol{p},-\boldsymbol{\pi})$.

In contrast, the transformed strategy used in Proposition 2 cannot be applied with $K>2$ users while assuring that the increasing differences property holds for every pair of users.

With logarithmic utility functions, it is shown in [5] that Problem P1 is a strictly concave maximization problem over the transformed variables $y_{k}=\log p_{k}$. In this case Problem P1 has a unique optimal solution, which is the only point satisfying the KKT conditions. Thus, $G_{F P P}$ has a unique NE corresponding to this optimal solution, and the ADP algorithm will converge to this point from any initial choice of powers and prices. With some minor additional conditions, the next proposition states that these properties generalize to other Type I utility functions.

Proposition 3: If for all $k \in \mathcal{K}, P_{k}^{\text {min }}>0$, and $G_{k}\left(\gamma_{k}\right) \in$ $[a, b]$ for all $\gamma_{k} \in\left[\gamma_{k}^{\min }, \gamma_{k}^{\max }\right]$, where $[a, b]$ is a strict subset of $[1,2]$, then Problem P1 has a unique optimal solution to which the ADP algorithm globally converges.

\section{CONCLUSIONS}

We have presented a distributed power control algorithm for SS wireless ad hoc networks. Each user announces a price, which is associated with the KKT conditions for the total utility maximization problem. This leads to an asynchronous power and price adaption algorithm, which requires only local knowledge of channel gains by each user. By interpreting the algorithm as myopic best response updates of a fictitious game, convergence can be proved with an appropriate strategy transformation using supermodular game theory. For users with logarithmic utilities, the algorithm globally converges to the unique socially optimal solution.

\section{REFERENCES}

[1] F. P. Kelly, A. Maulloo, and D. Tan, "Rate control for communication networks: Shadow prices, proportional fairness and stability," Journal of Operations Research Society, vol. 49, no. 3, pp. 237-252, March 1998.

[2] Y. Qiu and P. Marbach, "Bandwidth allocation in ad hoc networks: A price-based approach," in IEEE INFOCOM, 2003.

[3] P. Marbach and R. Berry, "Downlink resource allocation and pricing for wireless networks," in IEEE INFOCOM 2002, New York, NY, 2002.

[4] T. M. Heikkinen, "On congestion pricing in a wireless network," Wireless Networks, vol. 8, pp. 347-357, 2002.

[5] M. Chiang, "Balancing transport and physical layers in wireless multihop networks: Jointly optimal congestion control and power control,' IEEE Journal on Selected Areas in Communications, vol. 23, no. 1, pp. 104- 116, Jan 2005.

[6] D. M. Topkis, Supermodularity and Complementarity. Princeton University Press, 1998

[7] E. Altman, T. Boulogne, R. El-Azouzi, T. Jimenez, and L. Wynter, "A survey on networking games in telecommunications," Computers and Operations Research, in press.

[8] C. W. Sung and W. S. Wong, Lectures on Systems, Control and Information, Lectures at the Morningside Center of Mathematics. American Mathematical Society/International Press, 2000, ch. Mathematical aspects of the power control problem in mobile communication systems.

[9] C. Saraydar, N. B. Mandayam, and D. J. Goodman, "Efficient powe control via pricing in wireless data networks," IEEE Trans. on Communications, vol. 50, no. 2, pp. 291-303, Feb. 2002.

[10] E. Altman and Z. Altman, "S-modular games and power control in wireless networks," IEEE Trans. on Automatic Control, vol. 48, no. 5 , pp. 839-842, May 2003.

[11] T. Alpcan, T. Basar, R. Srikant, and E. Altman, "CDMA uplink power control as a noncooperative game," Wireless Networks, vol. 8, pp. 659670, 2002.

[12] N. Shroff, M. Xiao, and E. Chong, "Utility based power control in cellular radio systems," in IEEE INFOCOM, Anchorage, USA, 2001.

[13] T. Holliday, A. J. Goldsmith, , and P. Glynn, "Distributed power control for time varying wireless networks: Optimality and convergence," in Proceedings: Allerton Conference on Communications, Control, and Computing, 2003.

[14] D. Bertsekas, Nonlinear Programming, 2nd ed. Belmont, Massachusetts: Athena Scientific, 1999.

[15] A. Mas-Colell, M. D. Whinston, and J. R. Green, Microeconomic Theory. Oxford University Press, 1995.

[16] P. Milgrom and J. Roberts, "Rationalizability, learning and equilibrium in games with strategic complementarities," Econometrica, vol. 58, no. 6 , pp. 1255-1277, 1990. 